EESTI NSV TEADUSTE AKADEEMIA TOIMETISED. X KÖIDE

FOOSIKALIS-MATEMAATILISTE JA TEHNILISTE TEADUSTE SEERIA. 1961, NR. 4

НЗВЕСТИЯ АКАДЕМИИ НАУК ЭСТОНСКОЙ ССР. ТОМ X

СЕРИЯ ФНЗИКО-МАТЕМАТИЧЕСКИХ И ТЕХНИЧЕСКИХ НАУК. 1961, No 4

\title{
ОТВЕТ НА СТАТЬЮ П. КАРДА «ЕЩЕ РАЗ О ФОРМУЛАХ А. ВАШИЧЕКА»
}

АНТОНИН ВАШИЧЕК,

доктор физико-математических наук

Статья П. Карда [ㅁ], написанная в ответ на работу автора $\left[{ }^{2}\right]$, содержит ряд утверждений, с которыми нельзя согласиться.

П. Кард хочет, чтобы автор дал доказательство либо того, что формулы Мурмана противоречат теории Максвелла, либо того, что формулы автора не противоречат этой теории. В дальнейшем будет показано, что ни формулы Мурмана, ни формулы автора не противоречат теории Максвелла из-за двоякого толкования принципа обратнмости.

Недавно автор обратился к Мурману с просьбой сообщить ему теоретические основания того, что в формулах для отражения и прохождения света тонким слоем вместо вещественных показателей преломления стоят комплексные: Мурман коротко ответил, что в его работе «... теория остается в классических рамках, поэтому не следует ожидать ничего нового». Работа Мурмана - экспериментальная, и вывод своих формул он дает лишь в качестве введения без каких-либо теоретических рассуждений. Эти формулы вытекают из уровня знаний того времени. Если мы сегодня, с точки зрения новых знаний, видим недостатки теории Мурмана, то нашей обязанностью является их вскрытие, а не замалчивание.

Общеизвестно, и в книге автора [] тоже не раз подчеркивалось, что использованный ранее метод вывода, приводящий к так называемым простым формулам для отражения и прохождения света, верен только в случае, когда амплитуды отраженного и прошедшего света на первой границе слоя суть вещественные величины. В случае же отражения и пропускания света тонким металлическим слоем эти амплитуды комплексны, поэтому следует исходить из общих формул.

Кард возражает против того, что автор «применяет принцип обратимости и не дает никаких пояснений относительно его связи с теорией Максвелла». Это возражение сам Кард сразу же опровергает тем, что применяет принцип обратимости для доказательства правильности формул Мурмана при их выводе из теории Максвелла. Автор настоящей: статьи поэтому убежден, что Кард после проделанной им проверки применения метода для вывода формул Мурмана согласится как с принципом обратимости, так и с применением общих формул.

Следовательно, автор расходится с Кардом только в изложении принципа обратимости. Кард находит, что автор применяет принцип обратимости неправильно и что правильное применение принципа обра- 
тимости должно привести к формулам Мурмана. На утверждение Карда, что Власов применяет принцип обратимости только в непоглощающей среде, могу отметить, что по моим предположениям этот принцип, можно использовать и для системы диэлектрик-металл, если только ограничиться математическим разделом обеих сред.

Что касается применения принципа обратимости, то надо иметь в виду, что для комплексных амплитуд действительно существует два принщипа обратимости, отличающихся друг. от друга трактовкой и математической формулировкой. Ограничиваясь только первыми уравнениями этлх прннципов, назовем принцип, выражаемый формулой

$$
\mathrm{t}_{R}^{\prime} \mathbf{t}_{L}^{\prime}+\mathbf{r}_{R}^{\prime} \mathbf{r}_{R}^{\prime}=1
$$

а мплитудным, так как он относится только к амплитудам отражениюго и преломленного света; принцип же, данный формулой

$$
\mathbf{t}_{R}^{\prime} \mathbf{t}_{L}^{\prime \prime}+\mathbf{r}_{R}^{\prime} \mathbf{r}_{R}^{\prime *}=1 \text {, }
$$

назовем энергетическим, так как он относится к энергетическим состояниям на обенх сторонах раздела двух сред.

Рассмотрим более подробно оба эти принципа с точки зрения формул для отражения и пропускания света тонким слоем. Эти формулы можно вывести из граничных условий согласно теории Максвелла, но их можно вывести также из энергетических соотношений, например, как сумму комплексных амплитуд. В настоящее время в большинстве случаев применяют первый способ, но, по мнению автора, раньше (со времен Эри) преобладал второй метод вывода. Оба метода приводят к одинаковым результатам только в случае вещественных амплитуд. В случае же комплексных амплитуд результаты расходятся. Рассмотрнм формулы (1) и (2): является ли $\mathbf{r}_{R}^{\prime} \mathbf{r}_{R}^{\prime}$ квадратом комплексной амплитуды отраженного света, или же нужно брать согласно определению вектора Умова-Пойнтинга для комплексной величины произведенне $\mathbf{r}_{R}^{\prime} \mathbf{r}_{R}^{\prime}$, дающее интенсивность отраженного света? В том случае, когда в общчх формулах для отражения и пропускания света тонким слоем встречаются линейные амплитуды Френеля, подстановку комплексных величин считаем вполне однозначной. Но в случае квадратичных комплексных амплитуд вопрос состоит в том, взять ли квадрат комплексной амплитуды, или же произведение комплексно сопряженных амплитуд. Кард твердо убежден в том, что необходимо применять только амплитудный принцип обратимости. Но по мнению автора оба принципа обратимости следует рассматривать диалектически. Из-за двоякого принципа обратимости необходимо все заключения проверять численными данными и экспериментальными фактами. В этом вопросе мы принципиально расходимся с Кардом, который в теории Максвелла видит решение, не нуждающееся в проверке числовыми данными, опытом. Чисто теоретическое решение, однако, не может дать убедительногс ответа из-за двух возможных трактовок принципа обратимости. Следовательно, здесь нельзя обойтись без численной и экспериментальнй проверки.

Далее, Кард в своей статье ['] выводит формулы Мурмана с применением амплитудного принципа обратимости. Вполне естественно, что ограничиваясь амплитудными соотношениями для отраженного и преломленного света на границе диэлектрик-металл, нельзя получить чтолибо другое кроме формул Мурмана. Решение Карда служит лишь подтверждением этого простого решения. К формулам (8) и (9) Карда автор относится критически и, возможно, выскажет свое мнение позже. 
Что касается вопроса об энергетической проверке формул Мурмана, то автор вполне сознательно исходил только из энергетической проверки, но накопившийся в течение дальнейшего изучения материал свидетельствует против формул Мурмана еще более убедительно.

Мнение Карда о том, что неправильно связывать обсуждаемые формулы с тем, является ли слой сильно или слабо поглощающим, обусловлено тем, что он как теоретик решает эти вопросы исключительно теоретически без привлечения числовых данных. Автор же, как фнзик-экспернментатор, должен отстаивать требование экспериментальной проверки полученных формул. Если проделать расчеты отражательчой и пропускательной способностей тонкого металлического слоя по простой формуле для однократного отражения в слое (содержащей лишь первый член геометрической прогрессии) и по формуле Мурмана (учитывающей бесконечное множество отражений в тонком металлическом слое), то следовало бы ожидать, что отражательная способность, вычисленная по формуле Мурмана, будет больше или равиа отражательной способности, вычисленной по простой формуле. Но существуют такие пары величин $n_{1} \cdot$ и $n_{1} \kappa_{1}$ комплексного показателя преломления металлического слоя, при которых отражательная способность получается по формуле Мурмана меньше, чем по простой формуле, и можно также найти пары величин $n_{1}$ и $n_{1} \kappa_{1}$, для которых значения отражательной способности, вычисленные по обеим упомянутым формулам, приблизительно равны друг другу. Как в этих случаях объяснить, что сумма бесконечной геометрической прогрессии меньше ілли равна первому ее члену? Возражения автора против формул Мурмана исходят из сопоставления результатов вычисления отражательной способности тонкого металлического слоя (по различным формулам) с опыгными данными.

В заключение отметим, что значение дискуссии состояло в выяснении некоторых (быть может, не только для автора неясных) вопросов в өбласти теории отражения света тонким металлическим слоем. Целью автора было внести свой вклад в развитие этой области знания.

\section{Л И ТЕРА Т У Р А}

1. П. К а рд. Еще раз о формулах А. Вашичека, Изв. АН Эст. ССР, Серия физ,-мат. и техн. наук, т. X, № 4, 1961, 340 .

2. А. В а шичек, $\mathrm{K}$ теории отражения света тонким металлическим слоем, Изв. АН Эст. ССР, Серия физ.-мат. и техн. наук, т. IX, № 3, 1960, 242.

3. A. V a š i ček, Optics of Thin Films, North Holland Publishing Company, Amsterdam. 1960.
Университет г. Брно
Чехословацкая Соџиалистическая Республика
Поступила в редакцию 29. IX 1960

\section{3 аключительное $з$ самечание}

В ответ на статью профессора А. Вашичека могу ограничиться лишь сеылкой на свою предшествующую статью. Я согласен с профессором Вашичеком в том, что да.тьнейее ведение дискусси нецелесообразно. Оставаясь полностью при воззрения , высказанных в монх статьях (см. этот журнал за 1958 г., т. VII, № 4, стр. 283 и настояший выпуск стр. 340), счнтаю своим приятным долгом отметить плодотворность дискуссии.

\section{П. КАРД}

О т редакци и. На этом считаем дискуссию по вопросам теории тонкослойных покрытий законченной. 